\title{
Tree amplitudes at multiparticle threshold in a model with softly broken $O(2)$ symmetry
}

\author{
M.V. Libanov, V.A. Rubakov and S.V. Troitsky \\ Institute for Nuclear Research of the Russian Academy of Sciences, \\ 60th October Anniversary prospect, 7a, Moscow 117312
}

August 25, 2021

\begin{abstract}
Tree amplitudes of the production of two kinds of scalar particles at threshold from one virtual particle are calculated in a model of two scalar fields with $O(2)$ symmetric quartic interaction and unequal masses. These amplitudes exhibit interesting factorial and exponential behaviour at large multiplicities. As a by-product we observe that the kinematically allowed decay of one real particle into $n$ real particles of another kind, all at rest, has zero tree amplitude in this model for $n>2$.
\end{abstract}




\section{Introduction}

Recently, considerable attention have been paid to the study of multiparticle production processes both in the instanton sector [1, 2, 3, 4] and in the conventional perturbation theory [5, 6, 7, 8]. In the latter case, most of the results has been obtained in simple theories of one scalar field (or $p$ scalar fields with $O(p)$ symmetry), where a number of exact expressions have been derived, e.g., for the amplitudes of the production of $n$ particles at the multiparticle threshold by one virtual particle, at the tree level [07, 8, 9] and in the one loop approximation [10, 11, 12]. These amplitudes grow like $n$ ! at large $n$, and this non-trivial property is expected to persist to all orders of the perturbation theory [13, 14, 12].

For calculating the tree amplitudes at the threshold, two methods have been employed. One of them is based on recursion relations between diagrams with different numbers of final particles [7], and the other makes use of classical field equations with special boundary conditions [8]. Both of these methods have been generalized to incorporate loop corrections [10, 11, 12] and both work nicely in the simplest theories.

In this paper we study the tree amplitudes at the multiparticle threshold in the theory of two real scalar fields with the lagrangian

$$
L=\frac{1}{2}\left(\partial_{\mu} \varphi\right)^{2}+\frac{1}{2}\left(\partial_{\mu} \chi\right)^{2}-\frac{m_{1}^{2}}{2} \varphi^{2}-\frac{m_{2}^{2}}{2} \chi^{2}-\lambda\left(\varphi^{2}+\chi^{2}\right)^{2}
$$

The interaction term is $O(2)$-invariant, while the mass terms explicitly break $O(2)$ symmetry at $m_{1} \neq m_{2}$. This model may be viewed as a testing ground for different calculational techniques, and also has some features absent in the simplest theory of one scalar field. For example, the model contains an additional dimensionless parameter, $m_{1} / m_{2}$, and one can study the dependence of the amplitudes on this parameter. Also, the class of the tree amplitudes is wider than in the theory of one field because two kinds of particles can be emitted. Finally, in the case of broken 
reflection symmetry, $\varphi \rightarrow-\varphi$, there emerges a kinematically allowed possibility (at $\left.0>m_{2}^{2}>m_{1}^{2}\right)$ of a decay of an on-shell $\varphi$-particle at rest into $\chi$-particles, all of which are also at rest (of course, an interesting quantity is the amplitude of this decay and not the decay width which is zero because of empty phase space).

We find that the classical solution method [8] is convenient for the evaluation of the tree amplitudes at the threshold in the model (11). Due to a special symmetry of the corresponding classical equations [15], the explicit solution of the relevant boundary value problem can be found, and the explicit form of the tree amplitudes at particle threshold is obtained in this paper for the processes $\varphi \rightarrow n_{1} \varphi+n_{2} \chi$ and $\chi \rightarrow n_{1} \varphi+n_{2} \chi$, where the initial particle is off shell, for both unbroken $(<\varphi>=0$, $<\chi>=0)$ and broken $(<\varphi>\neq 0,<\chi>=0)$ cases. Not surprisingly, these amplitudes grow factorially at large $n_{1}$ and $n_{2}$, but besides the expected factorials, the amplitudes contain exponential terms depending non-trivially on $m_{\varphi} / m_{\chi}$ and $n_{1} / n_{2}$. Less obvious is the fact that the tree amplitudes of the decay of an on-shell $\varphi$-particle at rest into $n_{2} \chi$-particles with zero momenta (kinematically allowed in the spontaneously broken case) is zero for $n_{2}>2$.

In Sect.2 we obtain the general classical solution to the classical field equations for space-independent fields. In Sect.3 we evaluate the tree amplitudes at the threshold in the unbroken symmetry case $\langle\varphi>=<\chi>=0$. The broken symmetry case $<\varphi>\neq 0,\langle\chi>=0$ is considered in Sect.4. Sect.5 contains concluding remarks.

\section{Solution to classical equations}

We consider the tree amplitudes of, say, the processes $\varphi \rightarrow n_{1} \varphi+n_{2} \chi$, where the initial particle is off-shell and the final particles are on-shell and have zero spatial momenta. According to ref. [8], the generating function for these amplitudes, $\varphi_{c}\left(z_{1}, z_{2}\right)$, is the solution to the classical field equations for space-independent fields, obeying the con- 
dition that

$$
\varphi \rightarrow z_{1}+\varphi_{0}, \quad \chi \rightarrow z_{2}
$$

as $\lambda \rightarrow 0$, where

$$
z_{1}=a_{1} e^{i m_{\varphi} t}, \quad z_{2}=a_{2} e^{i m_{\chi} t}
$$

$m_{\varphi}$ and $m_{\chi}$ are the particle masses, $\varphi_{0}=\langle\varphi>$ (hereafter we assume $<\chi>=0$ ), and $a_{1}$ and $a_{2}$ are arbitrary constants. The amplitudes are then

$$
A_{n_{1}, n_{2}}=\left.\frac{\partial^{n_{1}}}{\partial z_{1}^{n_{1}}} \frac{\partial^{n_{2}}}{\partial z_{2}^{n_{2}}} \varphi_{c}\left(z_{1}, z_{2}\right)\right|_{z_{1}=z_{2}=0}
$$

Thus, we have to consider the hamiltonian for space-independent fields (we set the spatial volume equal to 1 ; the dependence on the volume can be easily restored),

$$
H=\frac{1}{2}(\dot{\varphi})^{2}+\frac{1}{2}(\dot{\chi})^{2}+\frac{m_{1}^{2}}{2} \varphi^{2}+\frac{m_{2}^{2}}{2} \chi^{2}+\lambda\left(\varphi^{2}+\chi^{2}\right)^{2}
$$

It has been found in ref.[15] that this hamiltonian possesses a non-trivial symmetry and thus is integrable. It has been also pointed out [16 that the system is separable in elliptic coordinates. So, we introduce new variables $\xi(t)$ and $\eta(t)$,

$$
\begin{aligned}
& \varphi=\sigma \sqrt{\left(\xi^{2}-1\right)\left(1-\eta^{2}\right)}, \\
& \chi=\sigma \xi \eta, \quad \sigma=\text { const. }
\end{aligned}
$$

By choosing

$$
\sigma^{2}=\frac{m_{1}^{2}-m_{2}^{2}}{2 \lambda}
$$

we find that the variables indeed separate in the Hamilton-Jacobi equation and we find for the truncated action $S(\xi, \eta)$,

$$
\begin{array}{r}
\left(\xi^{2}-1\right)\left(\frac{\partial S}{\partial \xi}\right)^{2}+2 \sigma^{2}\left\{\lambda \sigma^{4} \xi^{6}+\left(\frac{m_{1}^{2}}{2}-2 \lambda \sigma^{2}\right) \sigma^{2} \xi^{4}-\left(\frac{m_{1}^{2}}{2} \sigma^{2}-\lambda \sigma^{4}+E\right) \xi^{2}\right\}= \\
\left(\eta^{2}-1\right)\left(\frac{\partial S}{\partial \eta}\right)^{2}+2 \sigma^{2}\left\{\lambda \sigma^{4} \eta^{6}+\left(\frac{m_{1}^{2}}{2}-2 \lambda \sigma^{2}\right) \sigma^{2} \eta^{4}-\left(\frac{m_{1}^{2}}{2} \sigma^{2}-\lambda \sigma^{4}+E\right) \eta^{2}\right\}= \\
2 \sigma^{2} J
\end{array}
$$


where $E$ is the classical energy and $J$ is another integral of motion. Therefore, the truncated action is

$$
S=F(\xi)+F(\eta)
$$

where

$$
F(\xi)=\int d \xi \sqrt{\frac{2 \sigma^{2} J-2 \sigma^{2} \xi^{2}\left[\lambda \sigma^{4} \xi^{4}+\left(m_{1}^{2} / 2-2 \lambda \sigma^{2}\right) \sigma^{2} \xi^{2}-\left(m_{1}^{2} \sigma^{2} / 2-\lambda \sigma^{4}+E\right)\right]}{\xi^{2}-1}} .
$$

The action function is then

$$
S_{t o t}=-E t+F(\xi)+F(\eta)
$$

So, the general solution is determined by

$$
\begin{gathered}
\frac{\partial S_{t o t}}{\partial E} \equiv-t+\frac{\partial F(\xi)}{\partial E}+\frac{\partial F(\eta)}{\partial E}=-t_{0} \\
\frac{\partial S_{t o t}}{\partial J}=\beta
\end{gathered}
$$

where $\beta$ and $t_{0}$ are arbitrary constants. Thus, the general solution is finally obtained in the implicit form

$$
\begin{gathered}
\sigma^{2} \int d \xi \frac{\xi^{2}}{g(\xi)}+\sigma^{2} \int d \eta \frac{\eta^{2}}{g(\eta)}=t-t_{0} \\
\sigma^{2} \int d \xi \frac{1}{g(\xi)}+\sigma^{2} \int d \eta \frac{1}{g(\eta)}=\beta
\end{gathered}
$$

where

$$
g(\xi)=\sqrt{\xi^{2}-1} \sqrt{2 \sigma^{2} J-2 \sigma^{2} \xi^{2}\left[\lambda \sigma^{4} \xi^{4}+\left(\frac{m_{1}^{2}}{2}-2 \lambda \sigma^{2}\right) \sigma^{2} \xi^{2}-\left(\frac{m_{1}^{2}}{2} \sigma^{2}-\lambda \sigma^{4}+E\right)\right]}
$$

Since we are interested in complex solutions, the arbitrary constants $E, J, \beta$ and $t_{0}$ are also, in general, complex. We will see, however, that in both broken and unbroken symmetry cases, the relevant solutions correspond to $J=0, E=H(<\varphi>)=$ (vacuum energy), while $t_{0}$ and $\beta$ are determined by the conditions (2). 


\section{Unbroken reflection symmetry}

Let us first consider the case $m_{1}^{2}>0, m_{2}^{2}>0$ when $\langle\varphi\rangle \equiv \varphi_{0}=0$. To ensure the conditions (2) we note that in the limit $\lambda \rightarrow 0$, the fields $\varphi$ and $\chi$ do not interact with each other, and their energies, $E_{\varphi}$ and $E_{\chi}$, are the two integrals of motion. So, the integrals of motion of the non-linear problem, $E$ and $J$, may be expressed through $E_{\varphi}$ and $E_{\chi}$ in this limit. Obviously, $E=E_{\varphi}+E_{\chi}$, and it is straightforward to see from eq.(5) that $J=-E_{\chi}$ at $\lambda \rightarrow 0$.

For positive frequency solutions, eqs. (21) and (3), one has $E_{\varphi}=E_{\chi}=0$, so the relevant solution to the non-linear equations is determined by eqs. (6), (7) and (8) with

$$
E=J=0
$$

Then the system (6), (7) can be solved explicitly, and the solution obeying eqs. (2) and (3) is (see Appendix A for details)

$$
\begin{aligned}
& \varphi=z_{1}\left(1-\lambda \frac{\kappa}{2 m_{2}^{2}} z_{2}^{2}\right)\left(1-\frac{\lambda}{2 m_{1}^{2}} z_{1}^{2}-\frac{\lambda}{2 m_{2}^{2}} z_{2}^{2}+\lambda^{2} \frac{\kappa^{2}}{4 m_{1}^{2} m_{2}^{2}} z_{1}^{2} z_{2}^{2}\right)^{-1} \\
& \chi=z_{2}\left(1+\lambda \frac{\kappa}{2 m_{1}^{2}} z_{1}^{2}\right)\left(1-\frac{\lambda}{2 m_{1}^{2}} z_{1}^{2}-\frac{\lambda}{2 m_{2}^{2}} z_{2}^{2}+\lambda^{2} \frac{\kappa^{2}}{4 m_{1}^{2} m_{2}^{2}} z_{1}^{2} z_{2}^{2}\right)^{-1}
\end{aligned}
$$

where

$$
\kappa=\frac{m_{1}-m_{2}}{m_{1}+m_{2}}
$$

Notice that at $z_{2}=0$, the function (9) coincides with the known solution for one scalar field [8], as it should.

The amplitudes $\varphi \rightarrow n_{1} \varphi+n_{2} \chi$ are given by eq. (4). They are non-zero at

$$
\begin{gathered}
n_{1}=2 p+1, \\
n_{2}=2 q,
\end{gathered}
$$


where $p$ and $q$ are integer. We find

$$
A_{p, q}=\frac{(2 p+1) !(2 q) !}{m_{1}^{2 p} m_{2}^{2 q}}\left(\frac{\lambda}{2}\right)^{p+q} \sum_{l=0}^{\min (p, q)}(-1)^{l} \kappa^{2 l} \frac{(p+q-l-1) !}{l !(p-l) !(q-l) !}[p+q-\kappa q-(1-\kappa) l]
$$

The finite sum in eq.(11) can be transformed into the Jacobi polynomials of the argument $\left(1-2 \kappa^{2}\right)$ (see Appendix B), so that the expression for the amplitude takes the form

$$
\begin{gathered}
A_{p, q}=\frac{(2 p+1) !(2 q) !}{m_{1}^{2 p} m_{2}^{2 q}}\left(\frac{\lambda}{2}\right)^{p+q}(-1)^{p} \frac{p+q-1}{p q} \times \\
{\left[(p+q-\kappa q) P_{p-1}^{(1-q-p, 0)}\left(1-2 \kappa^{2}\right)-(q-1)(1-\kappa) \kappa^{2} P_{p-2}^{(2-p-q, 1)}\left(1-2 \kappa^{2}\right)\right]}
\end{gathered}
$$

An equivalent form of the amplitude can be obtained by transforming the sum in eq.(11) into the hypergeometric function F (see Appendix B). At $q \geq p$ we have

$$
\begin{gathered}
A_{p, q}=\frac{(2 p+1) !(2 q) !}{m_{1}^{2 p} m_{2}^{2 q}}\left(\frac{\lambda}{2}\right)^{p+q} \times \\
\left\{\kappa^{2 p}(-1)^{p} \frac{(q-1) !}{p !(q-p) !}\left[p F\left(-p, q ; q-p+1 ; \frac{1}{\kappa^{2}}\right)+(q-p)(1-\kappa) F\left(-p, q ; q-p ; \frac{1}{\kappa^{2}}\right)\right]\right\}
\end{gathered}
$$

while the expression at $q<p$ is obtained by interchanging $p \leftrightarrow q$ in the curly brackets in eq. (13).

Eq.(11) (or, equivalently, eqs.(12) or (13)) is the exact formula for the tree amplitudes. Its asymptotics at large $p$ and $q$ and $p / q=$ fixed has the form

$$
A_{p, q} \propto \frac{(2 p+1) !(2 q) !}{m_{1}^{2 p} m_{2}^{2 q}} \exp \left[(p+q) G\left(\frac{m_{1}}{m_{2}} ; \frac{p}{q}\right)\right]
$$

The explicit expression, given in Appendix $\mathrm{C}$, is not very illuminating. Notice that $A_{p, q}$ grows factorially as could have been expected.

A particularly simple asymptotics emerges at $p \rightarrow \infty, q \rightarrow \infty, p / q \rightarrow 1$. In that case we obtain (see Appendix C)

$$
A_{p, q} \propto \frac{(2 p+1) !(2 q) !}{m_{1}^{2 p} m_{2}^{2 q}}\left(\frac{\lambda}{2}\right)^{2 p}\left[\frac{\left(\sqrt{m_{1}}+\sqrt{m_{2}}\right)^{2}}{m_{1}+m_{2}}\right]^{2 p} C(p)
$$

where $C(p)$ depends also on $m_{1} / m_{2}$ and grows like some power of $p$. 


\section{Broken reflection symmetry}

At $m_{1}^{2}<0, m_{2}^{2}>m_{1}^{2}$, the symmetry $\varphi \rightarrow-\varphi$ is spontaneously broken, and

$$
<\varphi>\varphi_{0}=\frac{\left|m_{1}\right|}{2 \sqrt{\lambda}}
$$

The masses of excitations around this vacuum are

$$
m_{\varphi}=\sqrt{2}\left|m_{1}\right|, \quad m_{\chi}=\sqrt{\left|m_{1}\right|^{2}+m_{2}^{2}}
$$

The conditions (2) and (3) are satisfied when the integrals of motion are equal to

$$
J=0, \quad E=-\frac{m_{1}^{4}}{16 \lambda}=E_{v a c}
$$

The explicit solution to the equations of motion, that obeys the conditions (2) and (3), is obtained in the same way as in Sect.3. We find

$$
\begin{gathered}
\varphi=\varphi_{0}\left(1+\frac{z_{1}}{2 \varphi_{0}}+\frac{2 \lambda}{4 m_{\chi}^{2}-m_{\varphi}^{2}} z_{2}^{2}+\frac{\lambda}{\varphi_{0}} \frac{2 m_{\chi}-m_{\varphi}}{\left(2 m_{\chi}+m_{\varphi}\right)^{3}} z_{1} z_{2}^{2}\right) \times \\
\left(1-\frac{z_{1}}{2 \varphi_{0}}-\frac{2 \lambda}{4 m_{\chi}^{2}-m_{\varphi}^{2}} z_{2}^{2}+\frac{\lambda}{\varphi_{0}} \frac{2 m_{\chi}-m_{\varphi}}{\left(2 m_{\chi}+m_{\varphi}\right)^{3}} z_{1} z_{2}^{2}\right)^{-1} \\
\chi=z_{2}\left(1-\left(\frac{2 m_{\chi}-m_{\varphi}}{2 m_{\chi}+m_{\varphi}}\right) \frac{z_{1}}{2 \varphi_{0}}\right) \times \\
\left(1-\frac{z_{1}}{2 \varphi_{0}}-\frac{2 \lambda}{4 m_{\chi}^{2}-m_{\varphi}^{2}} z_{2}^{2}+\frac{\lambda}{\varphi_{0}} \frac{2 m_{\chi}-m_{\varphi}}{\left(2 m_{\chi}+m_{\varphi}\right)^{3}} z_{1} z_{2}^{2}\right)^{-1}
\end{gathered}
$$

The field (17) reduces to the known solution for spontaneously broken theory

of one scalar field [8] at $z_{2}=0$. On the other hand, eq.(18) does not coincide at $z_{1}=0$ with the solution for the theory of one field with unbroken symmetry [8]; in the diagrammatic language this corresponds to the existence of the diagrams of fig. $1 \mathrm{~b}$ absent in the theory of one field.

In the case of broken symmetry we should distinguish between the processes $\varphi \rightarrow$ $n_{1} \varphi+n_{2} \chi$ and $\chi \rightarrow n_{1} \varphi+n_{2} \chi$. The amplitudes of the former, which we denote by 
$A_{n_{1}, 2 q}^{\varphi}$ (the number of $\chi$-particles in the final state is even), are generated by $\varphi\left(z_{1}, z_{2}\right)$. The amplitudes of the latter, $A_{n_{1}, 2 q+1}^{\chi}$, are generated by $\chi\left(z_{1}, z_{2}\right)$. We find for the process $\varphi \rightarrow n_{1} \varphi+2 q \chi$

$$
\begin{gathered}
A_{n_{1}, 2 q}^{\varphi}=\frac{n_{1} !(2 q) !}{2 m_{\varphi}^{n_{1}-1}\left(4 m_{\chi}^{2}-m_{\varphi}^{2}\right)^{q}}(\sqrt{2 \lambda})^{2 q+n_{1}-1} \times \\
\sum_{l=0}^{\min \left(n_{1}, q\right)}(-1)^{l} \zeta^{2 l} \frac{\left(n_{1}+q-l-2\right) !}{l !\left(n_{1}-l\right) !(q-l) !}\left[\left(n_{1}+q-l-1\right)\left(2 n_{1}+2 q-3 l\right)+\left(n_{1}-l\right)(q-l) \zeta^{2}\right]
\end{gathered}
$$

where

$$
\zeta=\frac{2 m_{\chi}-m_{\varphi}}{2 m_{\chi}+m_{\varphi}}
$$

In analogy to Sect.3, the amplitudes in eq.(19) can be expressed through the Jacobi polynomials or hypergeometric function. We present here the latter form only, which reads

$$
\begin{gathered}
A_{n_{1}, 2 q}^{\varphi}=\frac{n_{1} !(2 q) !}{m_{\varphi}^{n_{1}-1}\left(4 m_{\chi}^{2}-m_{\varphi}^{2}\right)^{q}}(\sqrt{2 \lambda})^{2 q+n_{1}-1} \times \\
\left\{(-1)^{n_{1}} \zeta^{2 n_{1}} \frac{q !}{\left(q-n_{1}\right) ! n_{1} !}\left[F\left(-n_{1}, q+1 ; q-n_{1}+1 ; \frac{1}{\zeta^{2}}\right)-\frac{n_{1}}{q} F\left(-n_{1}+1, q ; q-n_{1}+1 ; \frac{1}{\zeta^{2}}\right)\right]\right\}
\end{gathered}
$$

Here it is assumed that $q \geq n_{1}$; at $q<n_{1}$ the expression for the amplitude is obtained by substituting $q \leftrightarrow n_{1}$ in curly brackets.

From the latter expression and eq.(30), the asymptotics of the amplitude at $n_{1} \rightarrow \infty, q \rightarrow \infty, n_{1} / q \rightarrow 1$ can be obtained,

$$
A_{n_{1}, 2 q}^{\varphi} \propto \frac{n_{1} !(2 q) !}{m_{\varphi}^{n_{1}-1}\left(4 m_{\chi}^{2}-m_{\varphi}^{2}\right)^{q}}(\sqrt{2 \lambda})^{2 q+n_{1}-1}\left[\frac{\left(\sqrt{2 m_{\chi}}+\sqrt{m_{\varphi}}\right)^{2}}{2 m_{\chi}+m_{\varphi}}\right]^{2 n_{1}} C\left(n_{1}, q\right)
$$

where $C\left(n_{1}, q\right)$ has power-like behaviour in this limit. The asymptotics in the general regime, $n_{1} \rightarrow \infty, q \rightarrow \infty, n_{1} / q=$ fixed, can also be found and has the general form of eq.(14).

From eq.(18) we find the amplitudes of the processes $\chi \rightarrow n_{1} \varphi+(2 q+1) \chi$, 


$$
\begin{gathered}
A_{n_{1}, 2 q+1}^{\chi}=\frac{n_{1} !(2 q+1) !}{m_{\varphi}^{n_{1}}\left(4 m_{\chi}^{2}-m_{\varphi}^{2}\right)^{q}}(\sqrt{2 \lambda})^{2 q+n_{1}} \times \\
\sum_{l=0}^{\min \left(n_{1}, q\right)}(-1)^{l} \zeta^{2 l} \frac{\left(n_{1}+q-l-1\right) !}{l !\left(n_{1}-l\right) !(q-l) !}\left[n_{1}+q-l-\zeta\left(n_{1}-l\right)\right]
\end{gathered}
$$

This expression can again be rewritten in terms of the Jacobi polynomials or hypergeometric function. At $n_{1} \rightarrow \infty, q \rightarrow \infty, n_{1} / q \rightarrow 1$ we have the following asymptotics,

$$
A_{n_{1}, 2 q+1}^{\chi} \propto \frac{n_{1} !(2 q+1) !}{m_{\varphi}^{n_{1}}\left(4 m_{\chi}^{2}-m_{\varphi}^{2}\right)^{q}}(\sqrt{2 \lambda})^{2 q+n_{1}}\left[\frac{\left(\sqrt{2 m_{\chi}}+\sqrt{m_{\varphi}}\right)^{2}}{2 m_{\chi}+m_{\varphi}}\right]^{2 n_{1}} C\left(n_{1}, q\right)
$$

We observe that both $A_{n_{1}, 2 q+1}^{\chi}$ and $A_{n_{1}, 2 q}^{\varphi}$ increase factorially at large number of final particles.

To conclude this section, we point out that at certain values of $\left|m_{1} / m_{2}\right|$ such that $m_{\varphi}=n_{2} m_{\chi}=2 q m_{\chi}$, there exists a kinematically allowed possibility of the decay of a $\varphi$-particle at rest into $n_{2}=2 q \chi$-particles, all of which are also at rest (according to eq.(16), this requires $0>m_{2}^{2}>m_{1}^{2}$ ). Eq.(19) tells us that this amplitude is in fact zero at the tree level, unless $n_{2}=2(q=1)$. Indeed, the amplitude $A_{0,2 q}^{\varphi}$ corresponds to the diagrams like those shown in fig.2, where the propagator of the initial particle is included. So, the decay amplitude $A_{\varphi \rightarrow 2 q \chi}^{\text {decay }}$ is obtained from $A_{0,2 q}^{\varphi}$ by truncating the leg corresponding to the initial particle,

$$
A_{\varphi \rightarrow 2 q \chi}^{\text {decay }}=\left(4 q^{2} m_{\chi}^{2}-m_{\varphi}^{2}\right) A_{0,2 q}^{\varphi}
$$

At $q=1$, the amplitude of eq.(19) has a pole at $m_{\varphi}^{2}=4 q^{2} m_{\chi}^{2}=4 m_{\chi}^{2}$, and one recovers the obvious result,

$$
A_{\varphi \rightarrow 2 \chi}^{\text {decay }}=-2 i \sqrt{2 \lambda} m_{\varphi}
$$

On the other hand, at $q>1\left(n_{2}>2\right)$, the amplitude of eq.(19) does not have a pole at $m_{\varphi}^{2}=4 q^{2} m_{\chi}^{2}$, and the decay amplitude (23) vanishes. This means, for example, that the diagrams of fig.2 with truncated initial legs, cancel each other for on-shell 
initial and final particles, all at rest (for the decay $\varphi \rightarrow 4 \chi$ this fact can of course be checked by the direct calculation of the diagrams).

\section{Conclusion}

The model of two scalar fields with $O(2)$ symmetric interaction and unequal masses has the property of integrability and separability of its classical equations for spatially homogeneous fields. This property enabled us to obtain the explicit formulas for the tree amplitudes at particle threshold for both unbroken and broken reflection symmetry $\varphi \rightarrow-\varphi$. These amplitudes are expressed through the Jacobi polynomials or, equivalently, finite hypergeometric series.

The behaviour of the tree amplitudes of the creation of $n_{1} \varphi$-particles and $n_{2} \chi$ particles at threshold at large $n_{1}, n_{2}$ and fixed $n_{1} / n_{2}$ is suggestive. It has the factorial growth and is exponential in the free parameters $m_{\varphi} / m_{\chi}$ and $n_{1} / n_{2}$,

$$
A_{n_{1}, n_{2}} \propto n_{1} ! n_{2} ! \lambda^{\left(n_{1}+n_{2}\right) / 2} \exp \left[\left(n_{1}+n_{2}\right) G\left(\frac{n_{1}}{n_{2}}, \frac{m_{\varphi}}{m_{\chi}}\right)\right]
$$

up to well defined powers of $m_{1}$ and $m_{2}$ and pre-exponential factors growing power-

like at large $n_{1}, n_{2}$. The function $G\left(\frac{n_{1}}{n_{2}}, \frac{m_{\varphi}}{m_{\chi}}\right)$ is calculable at the tree level; its explicit form at arbitrary $n_{1} / n_{2}$ in the unbroken case can be read off from the eq. (29), while at $n_{1} / n_{2} \rightarrow 1$ the corresponding expressions are particularly simple, see eqs. (15), (21) and (22).

Eq. (24) indicates that there may exist a semiclassical-type procedure for calculating the leading exponential behaviour of the amplitudes at large $n_{1}, n_{2}$. Such a procedure is explicit in the recent study [14] of the amplitudes in the theory of one scalar field with broken symmetry. So, one may anticipate that this feature is characteristic to a wide class of bosonic theories.

A peculiar property of the model with $\langle\varphi\rangle \neq 0$ is that the decay of a $\varphi$-particle 
into $2 q \chi$-particles at rest, although kinematically allowed at appropriate values of particle masses, has zero tree amplitude for $2 q>2$. This fact may or may not be related to the integrability of the classical theory of spatially homogeneous fields. We hope to discuss the cancellation of the corresponding diagrams (that occurs also for some other processes) in future.

The authors are indebted to D.T. Son, E.E. Tareyeva and P.G. Tinyakov for helpful discussions. One of us (V.R.) thanks M.B. Voloshin and L. McLerran for stimulating conversations at the initial stage of this work. The work of M.L. and S.T. is supported in part by the Weingart Foundation through a cooperative agreement with the Department of Physics at UCLA.

\section{Appendix A. Classical solution}

The integrals in eqs.(6), (77) are straightforward to evaluate at $E=J=0$. The system (6), (7) then takes the form

$$
\begin{gathered}
\frac{\left(\psi+m_{1}\right)\left(\mu+m_{1}\right)}{\left(\psi-m_{1}\right)\left(\mu-m_{1}\right)}=\exp \left[\mp 2 i m_{1}\left(t-t_{0}\right)\right] \\
\frac{\left(\psi+m_{2}\right)\left(\mu+m_{2}\right)}{\left(\psi-m_{2}\right)\left(\mu-m_{2}\right)}=\exp \left[\mp 2 i m_{2}\left(t-t_{0}-2 \beta \sigma^{2}\right)\right]
\end{gathered}
$$

where $\psi=\sqrt{a \xi^{2}+m_{2}^{2}}, \mu=\sqrt{a \eta^{2}+m_{2}^{2}}, a=m_{1}^{2}-m_{2}^{2}$. We note that the original fields $\varphi$ and $\chi$ can be expressed in terms of $\mu$ and $\psi$,

$$
\begin{aligned}
\varphi^{2} & =\frac{1}{2 a \lambda}\left(\psi^{2}-m_{1}^{2}\right)\left(m_{1}^{2}-\mu^{2}\right) \\
\chi^{2} & =\frac{1}{2 a \lambda}\left(\psi^{2}-m_{2}^{2}\right)\left(\mu^{2}-m_{2}^{2}\right)
\end{aligned}
$$

Therefore, to find the fields $\varphi$ and $\chi$ one does not need to evaluate $\xi(t)$ and $\eta(t)$, but one just has to solve the algebraic equations (25). This is easily done in terms of variables $(\psi \mu)$ and $(\psi+\mu)$. We find that the correct behaviour (2), (3) corresponds to the upper sign in eq. (25), and that the solution is given by eqs. (9), (10). 


\section{Appendix B. Reduction to Jacobi polynomials and hypergeometric functions}

The sum in eq.(11) can be rewritten in the following form,

$$
\begin{gathered}
\sum=\sum_{l} \frac{(p+q-1) !}{p ! q !}[(p+q-\kappa q)-(1-\kappa) l] \frac{\kappa^{2 l}}{l !} \times \\
\frac{[(-p+1) \cdots(-p+l)][(-q+1) \cdots(-q+l)]}{[-(p+q-1)+1] \cdots[-(p+q-1)+l]}
\end{gathered}
$$

This should be compared with the hypergeometric series 17, 18

$$
F(a, b ; c ; z)=\frac{\Gamma(c)}{\Gamma(a) \Gamma(b)} \sum_{l} \frac{\Gamma(a+l) \Gamma(b+l)}{\Gamma(c+l)} \frac{z^{l}}{l !}
$$

The direct comparison gives

$$
\begin{gathered}
\sum=\frac{(p+q-1) !}{p ! q !}\left[(p+q-\kappa q) F\left(-p+1,-q+1 ;-p-q+2 ; \kappa^{2}\right)-\right. \\
\left.(1-\kappa) \kappa^{2} \frac{(-p+1)(-q+1)}{(-p-q+2)} F\left(-p+2,-q+2 ;-p-q+3 ; \kappa^{2}\right)\right]
\end{gathered}
$$

Eq. (12) is then obtained by making use of the relations [17, 18] between the Jacobi polynomials and hypergeometric functions.

The representation (13) is obtained by changing the variable in the sum, $l \rightarrow p-l$, and then by using essentially the same trick.

\section{Appendix C. Asymptotics of the amplitudes}

Let us first evaluate the asymptotics of the Jacobi polynomials $P_{r}^{(\alpha, \beta)}(x)$ at $r \rightarrow \infty$, $\alpha \rightarrow-\infty, \alpha / r=-\theta=$ const. We make use of the generating function for the Jacobi polynomials,

$$
\mathcal{F}(y)=2^{\alpha+\beta} R^{-1}(1-y+R)^{-\alpha}(1+y+R)^{-\beta}
$$


where $R=\sqrt{1-2 x y+y^{2}},|y|<1$. The Jacobi polynomials are

$$
P_{r}^{(\alpha, \beta)}(x)=\left.\frac{d^{r} \mathcal{F}}{d y^{r}}\right|_{y=0}=\frac{1}{r !} \int \bar{y}^{r} \mathrm{e}^{-y \bar{y}} \mathcal{F}(y) d y d \bar{y}
$$

The integral in this equation is evaluated in the saddle point approximation. The saddle point is

$$
\begin{gathered}
\bar{y}=\frac{r}{y} \\
y=\frac{1}{4(\theta-1)}\left[-x(\theta-2)^{2}-\theta^{2}+(\theta-2)^{2} \sqrt{(x+1)\left(x+1+\frac{8(\theta-1)}{(\theta-2)^{2}}\right)}\right]
\end{gathered}
$$

In this way we obtain the asymptotics

$$
P_{r}^{(\alpha, \beta)}(x)=2^{\alpha} y^{r}(1-y+R)^{\alpha} C(r, \alpha)
$$

where $y$ and $R(y)$ are taken at the saddle point (27), and $C(r, \alpha)$ behaves like some power of $r$. Inserting eq. (28) into eq. (12) we obtain the asymptotics of the amplitude at

$$
\begin{gathered}
p \rightarrow \infty, \quad q \rightarrow \infty, \\
\theta=1+\frac{q}{p}=\text { fixed } \\
x=1-2 \kappa^{2}=\text { fixed }
\end{gathered}
$$

in the following form

$$
A_{p, q}=(-1)^{p} \frac{(2 p+1) !(2 q) !}{m_{1}^{2 p} m_{2}^{2 q}} q\left[\frac{\lambda(y q / p+1)}{8 \kappa^{2}(q / p-1)}\right]^{q+p} \frac{1}{y^{q}} C(p, q)
$$

where $C(p, q)$ behaves like a power of $p$ and $q, y(\theta, x)$ is given by eq. (27). Obviously, this expression has the form of eq. (14).

Eq. (29) looks singular at $p / q \rightarrow 1$, i.e. $\theta=2$. However, the singularity is, in fact, absent because $y=-1$ at that point. The asymptotics of the amplitude in this regime can be obtained either by the study of the integral in eq.(26) or by making use of eq. (13) and the appropriate asymptotics of the hypergeometric function. Namely, at $\Lambda \rightarrow \infty$ one has 19 


$$
F\left(\Lambda,-b-\Lambda ; c ; \frac{1}{\kappa^{2}}\right)=\frac{\Gamma(1+b+\Lambda)}{\Gamma(c+b+\Lambda)} \mathrm{e}^{\Lambda \xi} C(\Lambda)
$$

where

$$
\mathrm{e}^{\xi}=\frac{\kappa^{2}-2-2 \sqrt{1-\kappa^{2}}}{\kappa^{2}}
$$

and $C(\Lambda)$ behaves as some power of $\Lambda$ at $\Lambda \rightarrow \infty$. Eqs. (30) and (13) lead to eq. (15) in a straightforward way.

In this Appendix we did not discuss the pre-exponential factors in equations like eq. (28) or eq. (30). In principle, there could have occurred cancellations between different terms in eq. (12) or (13). We have checked by calculating the pre-exponentials that the cancellations occur in none of these formulas, i.e., the exponential behaviour, eqs. (14), (15) indeed takes place.

\section{References}

[1] A. Ringwald, Nucl. Phys. B330 (1990) 1.

[2] O. Espinosa, Nucl. Phys. B343 (1990) 310.

[3] M.P.Mattis, Phys. Rep. 214 (1992) 159.

[4] P.G. Tinyakov, Int. J. Mod. Phys. A8 (1993) 1823.

[5] J.M. Cornwall, Phys. Lett. 243B (1990) 271.

[6] H. Goldberg, Phys. Lett. 246B (1990) 445.

[7] M.B. Voloshin, Nucl. Phys. B383 (1992) 233.

[8] L.S. Brown, Phys. Rev. D 46 (1992) 4125. 
[9] E.N. Argyres, R.H.P. Kleiss and C.G. Papadopoulos, Nucl. Phys. B391 (1993) 42.

[10] M.B. Voloshin, Phys. Rev. D 47 (1993) R357.

[11] B.H.Smith, Summing one-loop graphs at multiparticle threshold, Univ. of Minnesota preprint TPI-MINN-92/50-T, 1992

[12] E.N. Argyres, R.H.P. Kleiss and C.G. Papadopoulos, Multiscalar amplitudes to all orders in perturbation theory, preprint CERN-TH.6852/93, 1993.

[13] M.B. Voloshin, Some properties of amplitudes at multi boson thresholds in spontaneously broken scalar theory, Univ. of Minnesota preprint TPI-MINN-92/61-T, 1992

[14] A.S. Gorsky and M.B. Voloshin, Non-perturbative production of multi-boson states and quantum bubbles, Univ. of Minnesota preprint TPI-MINN-93/20-T, 1993.

[15] R. Sahadevan and M. Lakshmanan, J. Phys. A 19 (1986) L949.

[16] M. Lakshmanan and R. Sahadevan, Phys. Rev. A 31 (1985) 861.

[17] I.S.Gradstein and I.M.Ryzhik, Table of Integrals, Series and Products, Academic Press, N.-Y., 1980.

[18] Handbook of Mathematical Functions, M.Abramowitz and I.Stegun, eds., Dover, N.-Y., 1964.

[19] H. Bateman and A. Erdélyi, Higher Transcendental Functions, vol.1, McGrawHill, N.-Y., Toronto, London, 1953. 


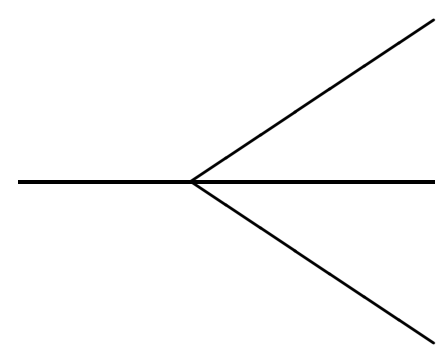

Fig.1a

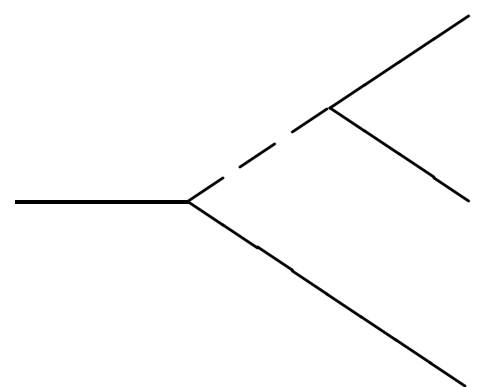

Fig. $1 b$

Figure 1: $\chi \rightarrow 3 \chi$ diagrams in the model with broken reflection symmetry $\varphi \rightarrow-\varphi$; solid and dashed lines correspond to the fields $\chi$ and $\varphi$, respectively.
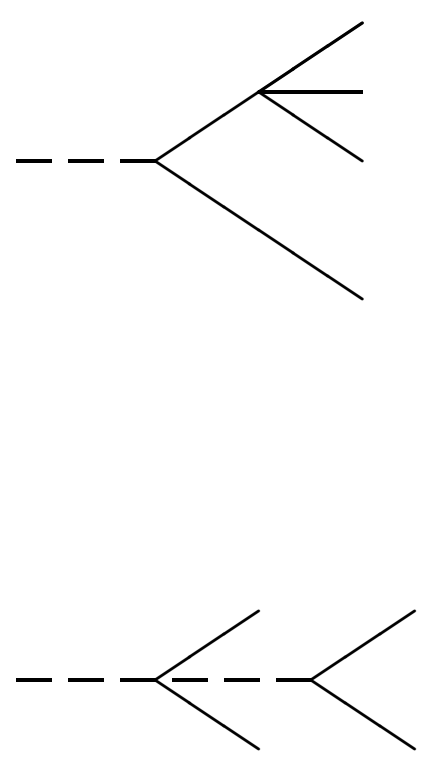
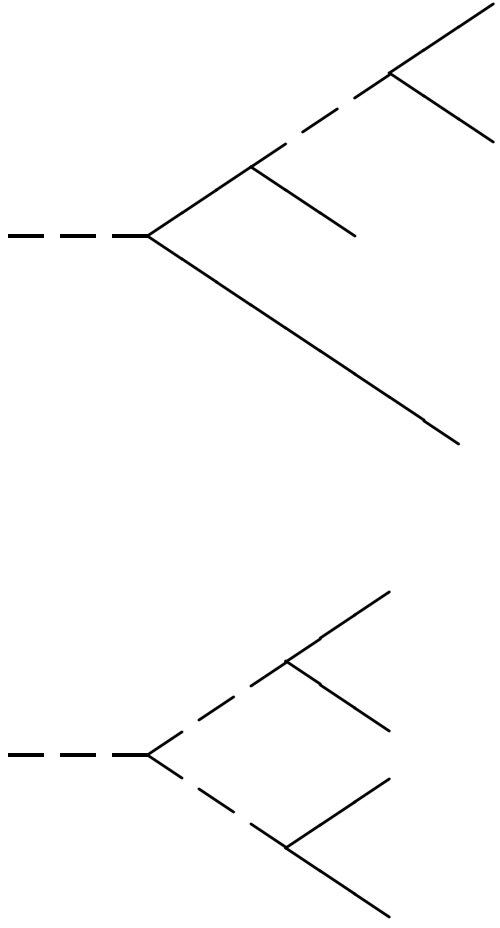

Fig.2

Figure 2: Diagrams for the decay $\varphi \rightarrow 4 \chi$ in the model with broken reflection symmetry. 